\title{
Desarrollo de la competencia comunicativa intercultural a través del trabajo con una obra literaria*
}

Development of the Intercultural Communicative Competence by Working with Literary Works Desenvolvimento da competência comunicativa intercultural através do trabalho com uma obra literária

Alexander Ramirez-Espinosa ${ }^{\text {a }}$

Universidad del Valle, Colombia

alexander.ramirez.e@correounivalle.edu.co

ORCID: http://orcid.org/0000-0002-7122-9537

Sol Colmenares-Rodríguez

Universidad del Valle, Colombia

ORCID: http://orcid.org/0000-0001-5806-0442

\section{Marcela Castellanos}

Universidad del Valle, Colombia

ORCID: http://orcid.org/0000-0002-5407-7180
DOI: https://doi.org/10.11144/Javeriana.syp38-74.dcci Redalyc: http://www.redalyc.org/articulo.oa $? \mathrm{id}=86059657001$

Fecha de recepción: 25 Junio 2018

Fecha de aprobación: 20 Febrero 2019

Fecha de publicación: 18 Junio 2019

\section{Resumen:}

Este artículo reporta una experiencia de creación de materiales para el abordaje de una obra literaria en un curso de inglés avanzado, y propone una reflexión sobre los elementos que se pueden tener en cuenta para la promoción y desarrollo de la competencia comunicativa intercultural (CCI) a partir de la literatura. Para tal fin se analizó un material compuesto por 84 actividades diseñado para guiar la lectura de la novela Americanah, de Chimamanda Adichie (2014). Las reflexiones a partir de tal análisis ponen de manifiesto la necesidad de que los docentes estudien con detenimiento sus prácticas de creación de materiales, de manera que identifiquen propósitos, posturas ideológicas implícitas y oportunidades pedagógicas para la promoción de la CCI.

Palabras clave: competencia comunicativa intercultural, literatura, diseño de materiales, lenguas extranjeras.

\section{Abstract:}

This article reports an experience of creating materials to approach a literary work in an advanced English course. It also proposes a reflection on the elements that can be considered in the promotion and development of the intercultural communicative competence (ICC) based on the literature. To do so, a material consisting of 84 activities designed to guide the reading of the novel Americanah by Chimamanda Adichie (2014) was analyzed. The reflections from this analysis expose the need that the teachers study in depth their creation of materials, in order to identify purposes, implicit ideological stances and pedagogical opportunities to promote the ICC.

Keywords: intercultural communicative competence, literature, design of materials, foreign languages.

\section{Resumo:}

Este artigo relata uma experiência de criação de materiais para abordar uma obra literária em um curso de inglês avançado e propõe uma reflexão sobre os elementos que podem ser levados em conta para promoção e desenvolvimento da competência comunicativa intercultural (CCI) a partir da literatura. Para Isso analisou-se um material composto por 84 atividades destinado a orientar a leitura do romance Americanah, de Chimamanda Adichie (2014). As reflexões a partir dessa análise põem de manifesto a necessidade dos docentes estudar com cuidado as suas práticas de criação de materiais, a fim de identificar propósitos, posturas ideológicas implícitas e oportunidades pedagógicas para a promoção da CCI.

Palavras-chave: competência comunicativa intercultural, literatura, desenho de materiais, línguas estrangeiras.

Notas de autor:

\footnotetext{
${ }^{a}$ Autor de correspondencia. Correo electrónico: alexander.ramirez.e@correounivalle.edu.co
} 


\section{Introducción}

El punto de partida de esta experiencia fue la decisión de tres profesores de inglés de diseñar e implementar una guía de trabajo para acompañar la lectura de la novela Americanah, de Chimamanda Adichie, en un curso de Tipologías orales en inglés. El objetivo principal fue proponer actividades que permitieran explorar la novela con distintos propósitos, y así crear dinámicas pedagógicas variadas. Más allá de esto, no hubo ninguna decisión predeterminada sobre la naturaleza de las consignas; sin embargo, una vez implementada la guía -y, en gran medida, estimulados por los temas que se abordan en la novela Americanab- se identificó un interés común entre los docentes por el tema del desarrollo de la CCI por medio del trabajo con una obra literaria.

Con esto en mente, este grupo de docentes decidió evaluar la guía producida y analizarla a la luz de la teoría existente en torno a la CCI y el diseño de materiales. El análisis permitió evidenciar patrones en el tipo de actividades planteadas, repeticiones en las consignas, excepciones y experimentos - tímidos algunos, avezados otros. Se encontraron también rastros de los preconceptos de los propios docentes y de sus ideologías que moldean, consciente o inconscientemente, las decisiones que se toman en la práctica pedagógica. Son estas reflexiones las que se presentan a continuación con el ánimo de contribuir a la discusión sobre el desarrollo de la competencia comunicativa intercultural en el aula de lenguas extranjeras, particularmente en relación con las posibilidades que ofrece la literatura.

\section{Referentes conceptuales}

\section{Literatura y competencia comunicativa intercultural}

Actualmente, se espera de quienes enseñan lenguas extranjeras que se promueva en las aulas el desarrollo de "una conciencia crítica de los valores y el significado de las prácticas en la cultura del otro así como en la propia” (Byram, 1997, p. 46 [traducción de los autores]). Esta conciencia hace parte, entre otros aspectos, de lo que se entiende en este campo por competencia comunicativa intercultural (CCI). Una persona competente interculturalmente tendrá la capacidad de interactuar con otras de diferentes orígenes e identidades, e incluso de servir de mediadora en situaciones conflictivas en las cuales sea necesario hacer uso de conocimientos preexistentes, de actitudes etnorrelativistas y de habilidades para descubrir, interpretar e identificarse con el otro (Byram, 1997).

$\mathrm{Al}$ preguntar cómo se puede incorporar a los currículos esta dimensión, nos encontramos con una gran aliada que puede convertirse en un puente hacia los sistemas de pensamiento que resultan ajenos; la literatura, esa ventana siempre abierta hacia la posibilidad de establecer contacto con otros lugares y otras épocas, puede ayudar a promover la reflexión intercultural entre los estudiantes. El texto literario, en tanto artefacto cultural, ofrece un lugar de interlocución en el que podemos identificarnos como criaturas emocionales, poner en escena historias personales y reconocernos como producto de un sistema de valores que aprendemos socialmente. Este ejercicio, fundamental para el desarrollo de la CCI, puede ser mejor aprovechado si se trae al aula "textos que reten a nuestros estudiantes en distintos niveles: por los temas provocativos que tocan, por la inclusión de personajes literarios antipáticos con los que puede ser difícil identificarse o la presencia de narradores cuya confiabilidad es dudosa" (Hoff, 2016, p. 64, [traducción de los autores]).

Entre las razones que se esgrime para usar literatura en la enseñanza de lenguas extranjeras, comúnmente se encuentra que los textos literarios son muestras genuinas de la lengua en uso, ya que ofrecen elementos para estimular la interacción en el aula y para desarrollar la conciencia lingüística, además de la posibilidad de analizar valores y actitudes. Asimismo, la lectura de obras literarias les permite a los estudiantes explorar la lengua en un contexto natural sin ninguna pretensión gramatical, los expone a sistemas culturales de distintas comunidades discursivas, y los lleva a la construcción de un pensamiento crítico. Esto último tiene que ver 
con la posibilidad de reflexión que representa la literatura, ya que los textos escritos, a diferencia de los orales, no requieren de respuestas inmediatas:

la naturaleza del diálogo entre lector y el texto es de alguna manera diferente, pues la palabra escrita invita al lector a un estilo de comunicación que es más deliberativo y reflexivo que el que se da en la interacción hablada”. (Hoff, 2016, p. 53, [traducción de los autores])

La combinación entre la experiencia lectora en solitario - que le da al estudiante autonomía para tomar decisiones en cuanto al ritmo de lectura y a sus necesidades individuales- y las actividades grupales en el aula de clase -que lo confrontan con las interpretaciones de los otros- generan una dinámica en la que la reflexión pausada y la negociación con el otro se alimentan mutuamente. Sin embargo, que un texto literario se convierta en un material educativo significativo para el aprendizaje depende en gran medida del uso que el docente haga de él y de las actividades que les proponga a sus estudiantes. El texto literario no promueve per se la interacción, tampoco expande la conciencia lingüística. Es el docente quien, al reconocerse y asumirse como agente cultural, puede hacer uso del potencial que el texto literario tiene. Si se quiere desarrollar una competencia intercultural en el aula, algunos textos tienen más potencial que otros. Entonces, ¿cómo identificarlos? Si estamos de acuerdo con Hoff en que los textos que traemos al aula deben retar a los estudiantes, un primer interrogante que se puede formular está relacionado con los temas que el texto aborda, las preguntas que se hace y las que suscita. Es nuestra convicción que en las aulas de lenguas extranjeras se tiene la obligación de hablar de temas incómodos, tales como la migración, la colonización y el refugio político, sólo por mencionar algunos. Todas estas "condiciones fronterizas" (Bhabha, 1994, p. 29), hermanas de la guerra, del exilio y del desplazamiento humano deben ocuparnos si nos interesa la denominada competencia comunicativa intercultural.

En este sentido, Tomlinson $(1998,2016)$ señala la necesidad de propender por actividades en las que los estudiantes se relacionen con textos complejos desde el punto de vista emocional y cognitivo, sin que la lengua sea el foco del ejercicio, que simula aquello que ocurre en nuestra lengua materna: "en nuestra primera lengua normalmente no estudiamos una obra literaria, sino que la experimentamos" (Tomlinson, 1998, p. 178 [traducción de los autores]). De manera similar, Rico-Troncoso (2012) está de acuerdo en que el aprendizaje intercultural implica un aprendizaje experiencial "en el que es necesario ser confrontado con situaciones desconocidas, experimentar inseguridad, temor, rechazo, así como seguridad, confianza y simpatía” (p. 142 [traducción de los autores]), lo cual se traduce en un trabajo a nivel emocional, perfectamente abordable desde la literatura.

Es nuestra labor, entonces, propiciar el uso de la literatura como una suerte de espejo en la que el estudiante se mira, se entiende y luego puede mirar al otro y entenderlo. Al respecto, Tomlinson (2000) comenta que la literatura "deja vacíos que el lector puede llenar con su propia experiencia, lo que hace que el lector sienta y reflexione al interactuar con el texto, haciendo uso de su experiencia de vida y, a la vez, agregando a ella" ( $\mathrm{p}$. 20 [traducción de los autores]).

La interacción con la otredad es inherente al uso de una lengua extranjera. La otredad, que nos conflictúa y nos cuestiona, nos obliga también a poner en escena una identidad desde y por la cual somos juzgados y juzgamos y que es permanentemente atravesada por el género, la etnia, la profesión, la edad y la orientación sexual; una identidad más o menos alfabetizada, más o menos subyugada, más o menos amenazada que se adapta, se esconde o se pone a la defensiva dependiendo de qué haya en juego en la interacción.

\section{Diseño de materiales}

El diseño y la adaptación de materiales son tareas recurrentes en la práctica del docente de lengua extranjera, no solo por el hecho de que ningún libro de texto cumple totalmente las expectativas y necesidades de docentes y aprendices (Núñez y Téllez, 2008) -y por ende recurrimos al uso de otros insumos que complementen 
nuestra propuesta didáctica-, sino también porque estas tareas estimulan el desarrollo de la creatividad, el pensamiento crítico y la conciencia sobre aspectos cognitivos, pedagógicos y didácticos sobre la lengua en los docentes que las asumen (Tomlinson, 2016). Por otra parte, teniendo en cuenta que los materiales constituyen la base fundamental del input que los estudiantes reciben en clase (Tomlinson, 2016), su desarrollo es una estrategia para garantizar una exposición a la lengua rica en aquellas características particulares que buscamos según el enfoque, los objetivos y las expectativas de enseñanza-aprendizaje que tenemos como maestros.

Así las cosas, si el desarrollo de la competencia comunicativa intercultural es un objetivo dentro de la clase de lengua extranjera, es estratégico e imprescindible el diseño de materiales tailored-made, es decir, hechos a la medida de un contexto particular y de los múltiples elementos que lo componen: nuestros estudiantes, su entorno cultural, sus preconcepciones, la lengua extranjera que están aprendiendo y las culturas asociadas a ésta, entre otros. Al respecto, Pulverness (2011) sostiene que los docentes tenemos "un rol vital de actuar como mediadores interculturales y proveer algunas de las coordenadas culturales que hacen falta en los libros de texto” (p.434 [traducción de los autores]). Esto último, por supuesto, se logra mediante el diseño de materiales que acompañen los demás recursos de aprendizaje usados en clase.

En esta misma línea, Rico-Troncoso (2011) afirma que los materiales son poderosas herramientas que facilitan el encuentro con la otra cultura y hacen a veces de puente para la mediación intercultural. Asimismo, los materiales que se diseñan en torno al desarrollo de la CCI "expanden las percepciones de los estudiantes sobre otras culturas y los empodera para desarrollar comportamientos como la tolerancia y el respeto hacia la diversidad" (Rico-Troncoso, 2011, p. 91 [traducción de los autores]).

Con esto en mente, el diseño de materiales apropiados en torno a textos literarios puede propiciar diversas reacciones en nuestros estudiantes, encaminadas hacia la toma de conciencia sobre sí mismos y sobre el otro, sobre las identidades propias y la otredad de diversos individuos, colectivos, pueblos y culturas. En este sentido, el diseño de materiales es una manera idónea de canalizar la riqueza literaria en beneficio del fomento de la $\mathrm{CCl}$ en nuestras clases.

\section{Descripción del contexto e instrumento de análisis}

\section{El curso}

Tipologías discursivas orales es un curso que se ofrece en el quinto semestre de la Licenciatura en Lenguas Extranjeras de la Universidad X. En el componente teórico se abordan distintas aproximaciones al tema de la oralidad, en relación con tradiciones retóricas, prácticas letradas y culturas. Es un curso en el que se enfatiza la comprensión auditiva y la comunicación oral en inglés, y en el que se busca continuar desarrollando la competencia comunicativa de docentes en formación a partir del aprendizaje basado en tareas y el aprendizaje cooperativo.

\section{La obra}

Americanah es una novela escrita por Chimamanda Ngozi Adichie en el año 2013, la cual cuenta la historia de Ifemelu, una ciudadana nigeriana quien parte a los Estados Unidos durante la dictadura militar de su país. En su condición de extranjera, experimenta por primera vez lo que implica ser una mujer afro, se enfrenta al racismo presente en la comunidad occidental y sufre transformaciones que ponen en jaque sus ideas sobre la identidad, el amor y su herencia cultural. Ifemelu documenta estos procesos de cambio en un blog, el cual representa para ella la posibilidad de hacer catarsis y de elaborar nociones nuevas sobre sus múltiples identidades: como mujer, como escritora, como extranjera y, sobre todo, como africana no americana. 


\section{La guía}

La guía de trabajo diseñada para acompañar la lectura de Americanah está compuesta por siete unidades, cada una de las cuales propone en promedio 12 ítems o ejercicios diferentes.

Por su extensión, no la incluimos dentro del artículo pero puede ser consultada en su totalidad en el siguiente link, https://drive.google.com/open?id=1lt4trIx5MovEep94bXYMTUH8-MudXa8I.

\section{El instrumento de análisis}

Con el fin de analizar las actividades propuestas en la guía, optamos por usar la matriz para evaluación de materiales diseñada por Rico-Troncoso (2012) (anexo 1). La matriz ofrece descriptores en cinco dimensiones.
1. Proficiencia (proficiency)
2. Conciencia (awareness)
3. Conocimiento (knowledge)
4. Actitud (attitude)
5. Habilidades (skills)

Los descriptores están enunciados en términos de las competencias que las actividades promueven.

\section{Análisis}

Después de pasar la guía por el tamiz de la matriz para la evaluación de materiales propuesta por RicoTroncoso (2011), se encuentran los porcentajes de frecuencia de actividades en cada dimensión, los cuales presentamos a continuación. Es importante notar que una misma actividad puede apuntar de manera simultánea al desarrollo de varias competencias en distintas dimensiones, de allí que cada porcentaje deba ser leído con respecto al total de las actividades analizadas. Teniendo este hecho en cuenta, el ejercicio arrojó los siguientes porcentajes (figura 1):

$100 \%$ de las actividades propuestas se enmarcan en la dimensión de Proficiencia.

$70 \%$ de las actividades propuestas se enmarcan en la dimensión de Conciencia.

$50 \%$ de las actividades propuestas se enmarcan en la dimensión de Conocimiento.

$30 \%$ de las actividades propuestas se enmarcan en las dimensiones de Actitud y Habilidades 


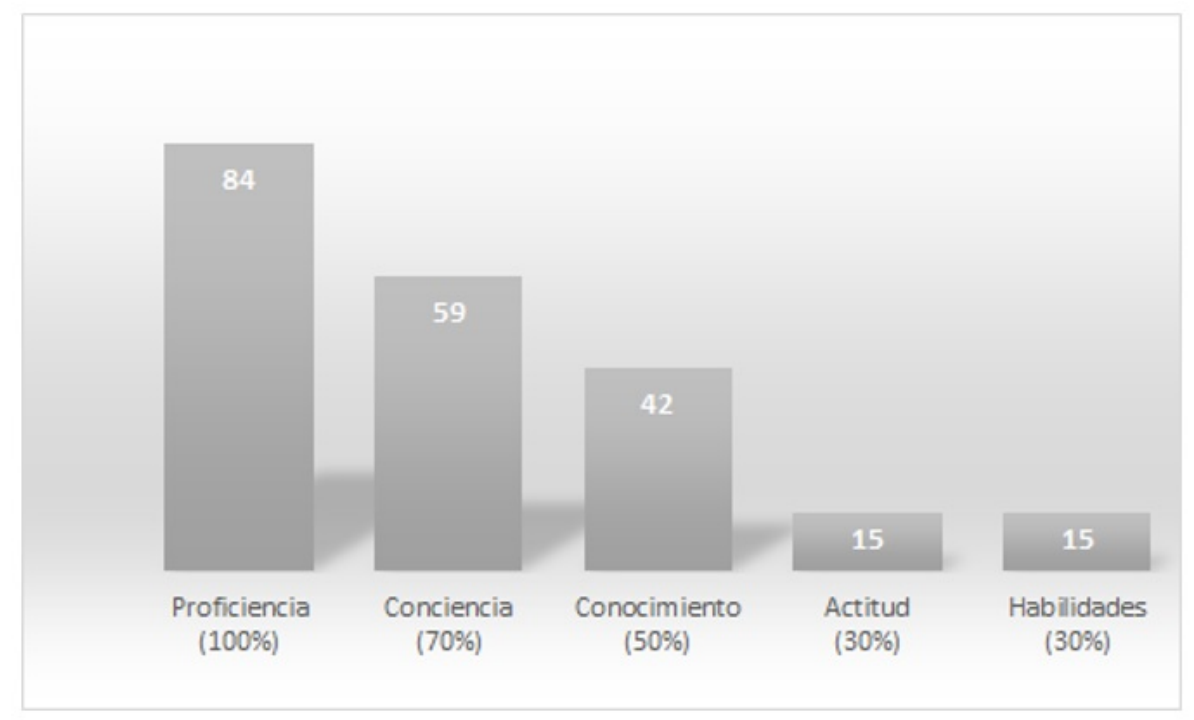

FIGURA 1

Análisis de las dimensiones propuestas por Rico-Troncoso (2011)

Fuente: elaboración propia

Las dimensiones de Attitude y Skills son las que presentan una menor presencia en el diseño de la guía, un promedio de $30 \%$ de las actividades analizadas están relacionadas con al menos uno de los descriptores en cada una de estas dimensiones. En el caso de Attitude, las actividades se centran, nuevamente, en el contraste entre culturas y el intercambio de opiniones sobre diversos temas. Solo dos actividades apuntan hacia evitar los juicios de valor y mensajes potencialmente ofensivos para la cultura extranjera. El descriptor en el que se presentó el menor número de actividades fue el relacionado con cómo nos vemos dentro de nuestra propia cultura, y la importancia de evitar juzgar a otros por sus hábitos alimenticios, su manera de vestir o su orientación sexual. De manera similar, en el caso de Skills no se observan actividades que apunten hacia demostrar flexibilidad en la interacción con personas de otras culturas, tal vez porque tales actividades exigen justamente la interacción real con extranjeros, lo cual puede ser limitado en el contexto del salón de clases.

Con respecto a la dimensión de Knowledge, encontramos que por lo menos el 50\% de las actividades analizadas propenden por contrastar la cultura propia con la extranjera, analizar comportamientos sociales apropiados e inapropiados, reflexionar sobre tabúes culturales y aspectos históricos, entre otros. Identificamos, por ejemplo, actividades que abordan problemas sociales y formulan interrogantes que "articulan la historia general con algunos factores sociopolíticos que han dado forma a la cultura del otro" (Rico-Troncoso, 2012, p. 153). Tal es el caso de la reflexión que se plantea sobre las relaciones entre "raza" y "clase", y sobre las visiones estereotipadas que median nuestras relaciones con otras personas. Mediante el análisis de citas textuales tomadas de la novela se propone, aunque de manera implícita, un ejercicio de hipertextualidad en el que se busca que el estudiante se interese por ahondar en el conocimiento de hechos históricos puntuales presentes en la novela, consultando diferentes fuentes o retroalimentándose de las discusiones con sus pares. Sin embargo, al igual que en la dimensión anterior, hubo un descriptor que no aparece reflejado en ninguno de los ejercicios propuestos, y se trata del reconocimiento de señales de estrés cultural.

La dimensión Awareness, junto con los ocho descriptores que la componen, es la segunda dimensión con mayor presencia en las actividades diseñadas. Aproximadamente el $70 \%$ de las actividades propuestas en las siete unidades estimulan el desarrollo de más de un descriptor a la vez. Un número importante de actividades apunta al reconocimiento de cómo percibimos las identidades culturales, y a la expresión de opiniones y puntos de vista sobre la cultura propia y la extranjera.

Identificamos que muchas de las actividades asociadas a esta dimensión le permiten al estudiante reconocer que el pensamiento es susceptible de ser transformado y que es posible cambiar gracias a la interacción con el 
otro; que es posible y deseable, en aras de la convivencia humana, moverse del eje de las propias creencias. Se percibe un consenso entre los profesores que diseñaron la guía en llevar a los estudiantes a estadios de reflexión sobre cómo el hecho de que algo o alguien nos resulte raro o extraño puede deberse a diferencias culturales. $\mathrm{Al}$ pedirle al estudiante que piense en cómo la lectura de la novela le ha permitido ver cosas que antes no veía, se abre un espacio para la introspección y, por ende, para el desarrollo de la conciencia. No obstante, no encontramos actividades que promovieran la reflexión sobre diferencias entre lenguas.

Predominan aquí las actividades de tipo comparativo, o las que proponen paralelos de similitudes entre el propio país y el país de donde viene el personaje principal, así como el paralelo entre maneras de reaccionar, en la cultura propia o en la foránea, frente a situaciones que aparecen en la novela. En general, se identificó un buen número de actividades (50\%, aproximadamente) enfocadas en reflexionar sobre cómo el contexto afecta/altera las interacciones con otras personas, cómo nos vemos dentro de nuestra propia cultura y cómo nos vemos en una cultura diferente. Prevalece una intención contundente de concientizar al estudiante sobre la naturaleza dinámica, variable y multifacética de los conceptos de cultura e identidad.

La dimensión más prominente a lo largo de todas las unidades es la de Proficiency. El 100\% de las actividades analizadas apuntan a la práctica lingüística o a la comunicación de temas concretos. No sorprende que, al tratarse de un material diseñado para un curso que se enfoca en el desarrollo de habilidades orales, las actividades promuevan la creación de escenarios para expresar y compartir opiniones y puntos de vista sobre diferentes temas, al tiempo que estimulan la práctica de estructuras y funciones de la lengua.

Llama la atención que una sola actividad promueva de manera explícita un ejercicio de comunicación usando lenguaje no verbal; sin embargo, en el resto de unidades y actividades el descriptor de uso de lenguaje no verbal no se identificó. Lo mismo ocurrió con el descriptor que promueve la comunicación de ideas de maneras distintas, con el cual sólo se asocia una actividad en la que se invita a los estudiantes a escribir una canción sobre los personajes y eventos de la historia, lo que les permite explorar un género diferente al original (narración) para la expresión. En cuanto al tipo de actividades asociadas al desarrollo de esta dimensión, se pudo observar una preferencia por las propuestas de reflexión a partir del análisis de citas específicas.

\section{Discusión}

\section{Sobre las actividades}

Que la Proficiencia sea la dimensión más prominente en las actividades propuestas nos hace pensar, por una parte, que, por ser el aspecto más concreto para propósitos evaluativos, los docentes nos vemos inclinados a incorporarla a las consignas con mayor frecuencia; y por otra, que la gramática y los aspectos formales de la lengua son los que más preocupan a los profesores. También es posible -ya que los descriptores en esta dimensión están enunciados en términos bastante generales (por ejemplo, practicar algunas características de la lengua y comunicarse sobre temas concretos) - que al hacer el análisis todas las actividades resulten incluidas en esta categoría.

La poca frecuencia de ejercicios que requieren el uso de lenguaje no verbal puede indicar que, o bien los profesores no lo consideran relevante, o no tienen conciencia de su pertinencia en la comunicación. La ausencia absoluta de actividades que promueven el reconocimiento de señales de estrés cultural llama particularmente nuestra atención ya que la novela plantea muchas situaciones en las que este tema podría ser explorado; de hecho, uno de los elementos más importantes en la trama es el choque cultural que el personaje principal sufre al dejar su país natal (Nigeria) para mudarse a los Estados Unidos. Asimismo, sorprende la poca presencia de consignas en las dimensiones de Actitud y Habilidades. Sobresale una preferencia de los docentes por aquellas actividades que estimulan la expresión de opiniones y puntos de vista sobre diferentes 
temas. Sin embargo, esto se hace desde la brecha que supone la abstracción nosotros vs. Ellos, y no desde una postura que articule las propias experiencias y contextos de vida.

En algunos casos, se propone el análisis de apartados específicos de la obra en el marco de unas categorías prediseñadas que de entrada sugieren el énfasis y tono de la discusión (por ejemplo: Language and Identity, Words and Culture, Perceptions about Education, Proverbs and Culture, Class and Identity, entre otros). Este marco permite guiar la atención de los estudiantes hacia el tipo de reflexión que se espera de ellos en sus interacciones.

Sumado a lo anterior, cuando pedimos a los estudiantes que confronten sus interpretaciones con las de sus compañeros y las del docente, estamos promoviendo una auténtica negociación de significados. Lo que sucede en el ejercicio solitario de lectura se convierte en material de análisis cuando se socializa en el aula. El solo hecho de descubrir que las interpretaciones de un texto pueden ser diferentes dependiendo de la experiencia lectora de cada uno puede conducirnos hacia el reconocimiento del significado como una construcción colectiva. Gadamer (citado en Hoff, 2016) describe la naturaleza de la interpretación como una forma de diálogo que transforma al intérprete como sujeto moral. La necesidad de interpretación surge cuando el sujeto es confrontado con un sistema de convicciones diferente al suyo. En esta construcción de significado, la perspectiva del otro amplía mi punto de vista y lo enriquece, un ejercicio nada fácil en las sociedades competitivas de las que hacemos parte, en las cuales "tener la razón" o imponer nuestros puntos de vista es considerado como una victoria.

Que la Conciencia sea la segunda dimensión más explorada en la guía nos hace pensar que las reflexiones sobre las diferencias entre culturas y sobre cómo percibimos las identidades son los aspectos más evidentes cuando hablamos de interculturalidad. Algo similar sucede con la dimensión de Conocimiento, en la cual contrastar la cultura propia con la extranjera es el ejercicio más frecuente. Cuando diseñamos actividades a partir de las cuales podemos comparar -sin dejar de reconocer nuestros prejuicios- comportamientos, creencias y significados de grupos humanos promovemos el respeto entre culturas. Estamos de acuerdo con Dos Reis Batista (citado en Bugnone, 2015) cuando advierte que en el contexto de enseñanza de una lengua extranjera "no se trata de que el aprendiz abandone su cultura para absorber otra y sí, de crear un puente de reflexión y aprendizaje de la cultura extranjera, respetando su propia cultura también” (p. 2).

A partir del diseño de materiales es posible promover en nuestros estudiantes un pensamiento crítico que les permita poner en cuestión su identidad cultural, es en ese cuestionamiento que se puede reconocer dicha identidad como un constructo polifacético en constante transformación. Como explicara Freire (2010), "en el fondo, no somos sólo lo que heredamos ni únicamente lo que adquirimos, sino la relación dinámica y procesal de lo que heredamos y lo que adquirimos" (p. 115).

De la misma manera en que, como hablantes de una lengua, modificamos nuestros registros de habla dependiendo de la situación comunicativa y de nuestros interlocutores, nuestro acervo cultural e intercultural nos permite tomar una postura, en una suerte de posicionamiento identitario, frente a otros sujetos. Se trata de un repositorio cultural que todos hemos construido a partir de nuestras experiencias de vida, pero del que tal vez no somos conscientes. En ese sentido, la literatura nos obliga a reconocer y enriquecer tal repositorio, de manera que nos volvemos más conscientes y críticos, y, por tanto, más competentes en la interacción. En nuestra experiencia particular en el curso, este uso de la literatura fue esencial en el proceso de ofrecer acceso a experiencias de choque cultural que no se podrían experimentar en un contexto inmediato.

La literatura nos permite cuestionar la homogeneidad que damos por sentada en la interacción con personas que reconocemos como pertenecientes a nuestra misma cultura. Nos permite experimentar extrañeza y la diversidad al interior del que reconocemos como nuestro propio grupo cultural, lo cual nos convence de que no existe una identidad monolítica, sino una construcción fluida, transformable, expandible. Siguiendo a Bhabha,

la diferencia cultural se convierte en un problema, no cuando puedes señalar (...) al punk con su cresta de siete pies de alto; ésta no tiene esa clase de visibilidad categórica. Es la extrañeza de lo familiar lo que resulta más problemático, tanto política como 
conceptualmente, cuando la diferencia cultural está en nosotros mismos como otros, en los otros como nosotros mismos, en esa zona fronteriza. (Bhabha citado en Gupta y Ferguson, 1992)

\section{Sobre el rol del docente}

En el desarrollo y promoción de la CCI, el docente transita entre el reconocimiento de sí mismo como sujeto cultural más o menos crítico, más o menos consciente, más o menos objetivo, con prejuicios y visiones estereotipadas, y la construcción de un sujeto mediador interculturalmente competente. Partiendo de esta idea, el docente tiene el doble reto de convertirse en mediador intercultural para guiar a sus estudiantes en esa misma dirección. Es en los estadios intermedios de ese continuum donde encontramos la pertinencia del diseño de materiales a partir de textos literarios como herramienta para el desarrollo de la CCI, y la necesidad de hacer de esos materiales objeto de investigación.

Estamos de acuerdo con Divine Che-Neba (2016) cuando afirma que los docentes estamos llamados a "ayudar a los estudiantes a dar forma a sus opiniones pre-existentes" (p. 178). De ahí la importancia de crear y mantener un ambiente de verdadera confianza en clase, donde exista la posibilidad de expresar abiertamente las creencias que tenemos y donde sea factible reconocer nuestros propios prejuicios, estereotipos, actitudes etnocéntricas y falta de empatía. Los docentes debemos ser un modelo en esta práctica. Presentarnos ante nuestros estudiantes como portadores de opiniones susceptibles de ser prejuiciosas puede hacernos sentir vulnerables, pero es el riesgo que necesitamos correr para dar lugar al cuestionamiento cotidiano del statu quo en nuestras prácticas de aula.

\section{Sobre la elección de textos literarios}

Después de analizar la guía diseñada para acompañar la lectura de la novela Americanah, reconocemos en esta obra literaria algunas características que bien pueden servir como elementos a tener en cuenta al seleccionar textos literarios con el propósito de desarrollar la competencia comunicativa intercultural. A continuación describimos tales características.

Americanab se inscribe en un canon literario anglófono que reconoce y legitima la diversidad étnica y las identidades en conflicto. La novela nos ofrece, tal como lo expresa Divine Che-Neba en su artículo Culture and Identity: Towards Redefining Representational Material for the ESL/EFL Classroom, miradas "del mundo Africano-Americano, Americano-Africano, Africano-Británico, del Africano en Nigeria y de los Africanos en la diáspora en general con su herencia e historia de violencia, subyugación, explotación, abuso y segregación" (2016, p. 175). Toda esta diversidad nos permitió explorar, no solo el reconocimiento de variedades léxicas y estrategias discursivas, sino también, y sobre todo, la discusión de temas tales como relaciones de poder, construcción de identidades y conflicto. Temas pertinentes para la creación de prácticas pedagógicas en las cuales nuestros estudiantes puedan observar al otro desde la empatía que genera el reconocerle como ser humano condicionado por el sistema económico, social y político en el que ha crecido. La literatura que pone en la mira el tema de la migración, desde el punto de vista del que migra, es a nuestro juicio un engranaje para generar ese movimiento que nos lleva a salir de la zona de confort en la cual el "nosotros" y el "ellos" son entidades delimitadas, fácilmente identificables.

Otra característica de Americanah que se hizo evidente luego del análisis es que nos ofrece muestras de distintos géneros discursivos; cartas, diarios y entradas de blog son algunos de ellos. Consideramos que se hubiera podido aprovechar más esta característica de la obra, teniendo en cuenta que un insumo de este tipo puede ser usado para el diseño de actividades que contemplen el reconocimiento de la estructura retórica, las condiciones de enunciación y las intenciones con las que se producen y se ponen en circulación este tipo de géneros. Entender que los textos no son inocentes y que responden a relaciones de poder que se gestan y se 
actualizan en las interacciones de sujetos culturales hace parte de la comprensión del papel determinante de la cultura en las producciones textuales, su circulación y posterior reconocimiento o censura.

La novela también es rica en reflexiones sobre vocabulario desde una perspectiva que trasciende lo gramatical. La autora pone en boca de Ifemelu, el personaje principal, cuestionamientos que la llevan a indagar sobre el peso histórico de términos controversiales y sobre los juicios que sopesan los hablantes al escoger o evitar una variedad léxica o dialectal.

Resulta pertinente, entonces, escoger obras literarias que exploren puntos de vista disidentes, temáticas transgresoras, autores de procedencias diversas, de comunidades minoritarias y minorizadas, con visiones del mundo particulares que reten los tabúes normativos convencionales. La lengua le pertenece al que la usa y, por lo tanto, es válido poner las lenguas extranjeras que enseñamos en boca del no nativo, del hablante inmigrante, del oprimido, del marginal y, en general, de todo aquel que tenga una historia que nos confronte.

\section{A manera de conclusión}

$\mathrm{Al}$ indagar en el papel del docente universitario como diseñador de materiales, particularmente para el desarrollo de la competencia comunicativa intercultural, concluimos que vale la pena hacer de los materiales que diseñamos, un objeto de investigación. El ejercicio de mirar en retrospectiva, de poner bajo la lupa nuestras decisiones y reflexionar sobre las razones que las motivan, nos permite reconocer preconceptos e identificar cómo opera la ideología en nuestras prácticas pedagógicas. Se evidencia mediante este ejercicio qué buscamos como docentes, a qué le damos prioridad y qué dejamos de lado. Aquí emergen nuestros temores hacia lo que nos resulta nuevo y nuestra tendencia a quedarnos en la dimensión que consideramos más "segura" para las prácticas evaluativas: la proficiencia.

En esa mirada retrospectiva resulta muy útil la matriz propuesta por Rico-Troncoso (2012), cuya versatilidad quedó validada a lo largo de esta experiencia. Las dimensiones propuestas, así como los descriptores que el autor propone para su análisis, permiten clasificar las actividades que los docentes diseñamos, ofrecen lineamientos que se pueden incluir en el diseño de actividades y materiales para la promoción de la CCI, y pueden ser usadas como criterios para la selección de obras literarias ricas en elementos interculturales.

A continuación, describimos algunos criterios que pueden resultar útiles al escoger un texto literario con el propósito de desarrollar la CCI a nivel universitario. Primero, consideramos esencial analizar la obra en términos de los temas que aborda. Los textos que ofrecen miradas sobre la diversidad étnica, la construcción y transformación de las identidades y los contextos económicos, políticos y sociales que les dan forma son terrenos fértiles para el desarrollo de la conciencia y el conocimiento, dos dimensiones centrales de la CCI. En segundo lugar, cabe preguntarse si la obra ofrece elementos para cuestionar la producción y circulación de diversos géneros discursivos, ya que, como mencionamos anteriormente, los textos se construyen bajo condiciones sociales, políticas y económicas concretas, y se gestan en las intenciones específicas del autor. El tercer criterio que reconocemos como útil después del análisis está relacionado con el manejo que se le da al vocabulario en el texto. Para aproximarse a la semántica del texto desde una perspectiva intercultural, es relevante preguntarse si hay reflexiones en la obra en torno al léxico y registro de los personajes, así como cuestionar si el texto ofrece elementos para abordar discusiones sobre las motivaciones que tienen los hablantes y las implicaciones que dicha escogencia trae consigo.

Aunque parezca obvio, no podemos perder de vista que además de los criterios que aquí recomendamos, la escogencia de la obra se realiza teniendo en cuenta los objetivos particulares del curso, los contenidos que se deben trabajar y el desarrollo de la competencia intercultural. Ahora bien, si nos interesa posicionar a nuestros estudiantes como interlocutores en la reflexión pedagógica, es de suma importancia socializar con ellos el proceso de selección. Explicitar las razones por las que se escoge la obra redundará en una mejor comprensión 
de las actividades propuestas por el profesor. Finalmente, concluimos que la literatura es una herramienta poderosa para el desarrollo de la competencia comunicativa intercultural, desde una perspectiva que entiende la identidad como una construcción que se nutre de las interacciones con otros y de las reflexiones que surgen a partir de esas experiencias.

\section{Referencias}

Adichie, C. (2014). Americanah. Nueva York: Anchor Books.

Bhabha, H. K. (1994). El lugar de la cultura. Buenos Aires: Manantial.

Bugnone, A. (2015, junio). Lengua, cultura e interculturalidad en la enseñanza de lenguas extranjeras. Conferencia presentada en VIII Coloquio PELSE, Santa Fe, Argentina. Recuperado de http://www.memoria.fahce.unlp.ed u.ar/trab_eventos/ev.4124/ev.4124.pdf

Byram, M. (1997). Teaching and Assessing Intercultural Communicative Competence. Multilingual Matters. Clevedon: Multilingual Matters.

Che-Neba, D. (2016). Culture And Identity: Towards Redefining Representational Material For the ESL/EFK Classroom. Asian Journal of Social Sciences \& Humanities, 5(2), 172-179.

Freire, P. (2010). Cartas a quien pretende enseñar (2.a ed.). Buenos Aires: Siglo Veintiuno Editores.

Gupta, A., y Ferguson, J. (1992) Space, Identity and the Politics of Difference. Cultural Anthropology, 7(1), 6-23.

Hoff, H. E. (2016). From 'Intercultural Speaker' to 'Intercultural Reader': A Proposal to Reconceptualize Intercultural Communicative Competence Through a Focus on Literary Reading. En F. Dervin y Z. Gross (eds.), Intercultural Competence in Education (pp. 51-71). Londres, Reino Unido: Palgrave Macmillan.

Núñez, A., y Téllez, M. (2008). Meeting students' needs. Enletawa Journal, 1, 65-68.

Pulverness, A. (2011). Materials for Cultural Awareness. En B. Tomlison (ed.), Developing Materials for Language Teaching (pp. 426-438). Londres, Inglaterra: Continuum.

Rico-Troncoso, C. (2011). The effects of language materials on the development of intercultural competence. En B. Tomlinson y H. Masuhara (eds.), Research for Materials Development in Language Learning (pp. 83-102). Londres, Inglaterra: Continuum.

Rico-Troncoso, C. (2012). Language teaching materials as mediators for ICC development: a challenge for materials developers. Signo y Pensamiento, 31(60), 130-154.

Tomlinson, B. (1998). And now for something not completely different: an approach to language through literature. Reading in a Foreign Language, 11(2), 177-189.

Tomlinson, B. (2000). Materials for cultural awareness: Combining language, literature and culture in the mind. The Language Teacher, 24(2), 19-21.

Tomlinson, B. (2016). The Importance of Materials Development for Language Learning. En M. Azamoosh, M. Zeraatpishe, A. Faravani y H. Reza (eds.), Issues in Materials Development (pp. 1-9). Rotterdam, Países Bajos: Sense Publishers.

\section{Notas}

* $\quad$ Artículo de reflexión

Licencia Creative Commons CC BY 4.0

Cómo citar este artículo: Ramírez-Espinosa, A., Colmenares-Rodríguez, S., y Castellanos, M. (2019). Desarrollo de la competencia comunicativa intercultural a través del trabajo con una obra literaria. Signo y Pensamiento, 38(74). https://doi.org/10.11144/Javeriana.syp38-74.dcci 


\section{Anexo 1}

\begin{tabular}{|c|c|c|c|}
\hline \multicolumn{4}{|c|}{ INTERCULTURAL COMMUNICATIVE COMPETENCE AND MATERIALS DEVELOPMENT } \\
\hline DIMENSIONS & DESCRIPTORS & Yes & No \\
\hline \multirow{5}{*}{ AWARENESS } & Differences across languages & & \\
\hline & Differences across cultures & & \\
\hline & How context affect/alter interactions with others & & \\
\hline & How she/he views her/himself within her/his own culture & & \\
\hline & How she/he views her/himself within a different culture & & \\
\hline \multirow{3}{*}{$\begin{array}{l}\text { In this activity, } \\
\text { students can } \\
\text { reflect on... }\end{array}$} & $\begin{array}{l}\text { How she/he perceives different cultural identities (race, class, gender, } \\
\text { age, ability) }\end{array}$ & & \\
\hline & Respecting multiple and different viewpoints & & \\
\hline & Social issues (weather changes, poverty, food crisis, etc) & & \\
\hline \multirow{5}{*}{ ATTITUDE } & Value his/her own culture & & \\
\hline & Value the host culture & & \\
\hline & Express his/her own opinions and views about different subjects & & \\
\hline & Express his/her needs & & \\
\hline & Express his/her wants & & \\
\hline \multirow[t]{2}{*}{$\begin{array}{l}\text { In this activity, } \\
\text { students can... }\end{array}$} & $\begin{array}{l}\text { Avoid making judgments to different cultures (food, dress, sexual } \\
\text { orientation) }\end{array}$ & & \\
\hline & $\begin{array}{l}\text { Avoid sending offending messages to different cultures (food, dress, } \\
\text { sexual orientation) }\end{array}$ & & \\
\hline
\end{tabular}

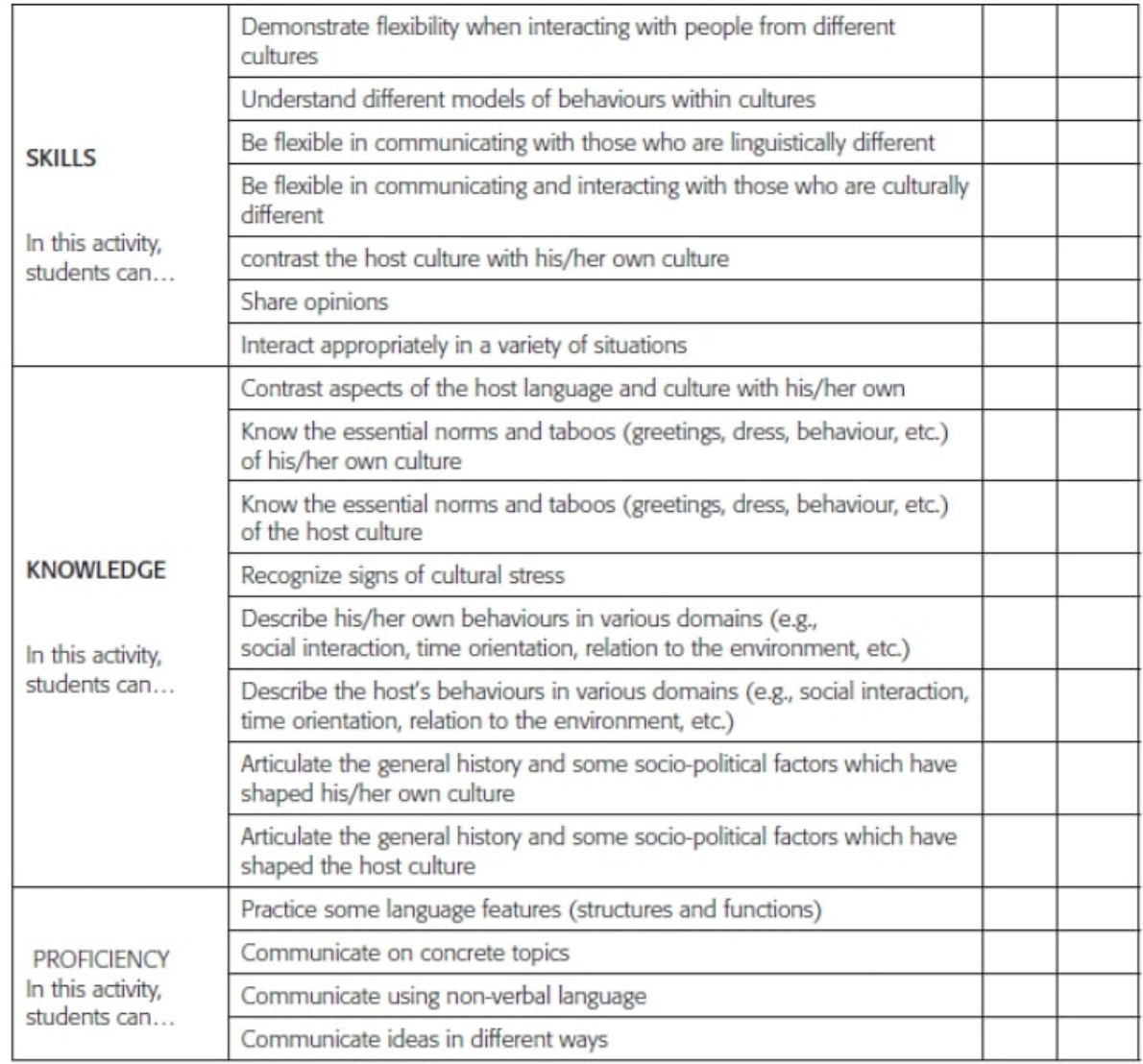

Matriz para la evaluación de materiales Fuente: Rico-Troncoso (2012) 